\title{
Modeling Metamaterial Transmission Lines loaded with Pairs of Coupled Split Ring Resonators
}

\author{
Lijuan Su, Jordi Naqui, Student Member, IEEE, Javier Mata-Contreras, Ferran Martín, Fellow, IEEE
}

\begin{abstract}
A lumped element equivalent circuit model of the unit cell of metamaterial transmission lines loaded with pairs of coupled split ring resonators (SRRs) is presented. It is assumed that the dominant coupling mechanism between the SRRs forming the pair is magnetic, and that the distance between SRRs of adjacent cells is high enough to neglect such additional inter-resonator coupling. SRRs are oriented with their symmetry plane orthogonal to the line axis. Under these conditions, the line-to-SRR coupling is also magnetic, the electric coupling being negligible. The presented model accounts for the rupture of symmetry that can be caused, for instance, by asymmetric dielectric loading of the SRRs. Thus, the analysis is carried out on a general model where the SRRs of the pair have different inductance and capacitance. Then, different cases are studied, in particular a line with identical SRRs, and a line with different SRRs, but with the same resonance frequency. It is shown that coupling between SRRs tends to far or split the resonance frequencies of the loaded lines (transmission zeros), except for the symmetric case, where only one resonance (different to the one of uncoupled SRRs) appears. The model is validated by comparing circuit simulations using extracted parameters with electromagnetic simulations and experimental data.
\end{abstract}

Index Terms- Electromagnetic metamaterials, metamaterial transmission lines, split ring resonators (SRRs).

\section{INTRODUCTION}

A rtificial transmission lines inspired by metamaterial concepts, also called metamaterial transmission lines, have been a subject of intensive research in the last years. These lines are implemented by loading a host line with reactive elements, including inductors, capacitors and/or resonators. The main relevant aspect of metamaterial transmission lines, as compared to ordinary lines, is the fact that, thanks to the presence of the reactive elements, there are more degrees of freedom for design purposes. It is thus possible to tailor both the characteristic impedance and the dispersion, opening the path to the implementation of microwave components with novel functionalities on the basis of impedance and dispersion engineering [1]. To a first order approximation, ordinary lines are dispersionless. Hence, dispersion engineering is the main differential (and genuine) aspect of metamaterial transmission lines as compared to their conventional counterparts. Enhanced bandwidth components [2]-[5], multiband components [6]-[8], and leaky wave

This work has been supported by MINECO (Spain) under projects TEC201017512, TEC2013-40600-R and CSD2008-00066, and AGAUR-Generalitat de Catalunya under projects 2009SGR-421 and 2014SGR-157. Jordi Naqui is in debt to MECD (Spain) for supporting his work through the FPU grant AP2010-0431. Ferran Martín is in debt to ICREA for supporting his work.

The authors are with GEMMA/CIMITEC, Departament d'Enginyeria Electrònica, Universitat Autònoma de Barcelona, 08193 Bellaterra, Spain (email: Ferran.Martin@uab.es). antennas with end-fire to back-fire scanning capability [9][11] are some of the proposed microwave components where dispersion engineering plays a fundamental role in their designs.

Many of the previous metamaterial based components have been implemented by means of the so-called resonant-type approach [12], where the host line is loaded with split ring resonators (SRRs) [13] or with other related resonant particles (such as CSRRs [14],[15]). For design purposes, lumped element equivalent circuit models of the unit cells of these artificial lines are very useful. This paper is focused on the first approach for the implementation of resonant-type metamaterial transmission lines: coplanar waveguides loaded with pairs of SRRs [13]. These lines exhibit a stop band functionality that may be interpreted as due to the extreme effective permeability in the vicinity of the fundamental SRR resonance (positive below it and negative above it). By loading these lines with shunt inductive strips, the stop band is switched to a pair of pass bands with composite right/left handed (CRLH) behavior, namely, backward wave propagation at the lower transmission band (due to the combined effect of SRRs and shunt strips), and forward wave propagation at the upper transmission band (caused by the host CPW) [16].

The first model of these CPWs loaded with SRRs and shunt strips was reported in [13], and later revised in [16] to account for the effects of the inductive strips on the transmission zero frequency, present below the first transmission band. In these models, coupling between SRRs of adjacent cells was not included, and the orientation of the SRRs was considered to be with their symmetry plane orthogonal to the line axis. With such orientation, the single coupling mechanism between the line and the SRRs is magnetic. However, by rotating the SRRs electric coupling must also be accounted for in the model (giving rise to mixed coupling), and it explains the asymmetry in the reflection coefficients measured from the two ports, as demonstrated in [17]. Coupling between adjacent SRRs was considered in [18],[19]. In [19] it was demonstrated that stopband bandwidth enhancement in SRRloaded CPW transmission lines is due to the presence of complex modes [20], which appear as conjugate pairs and do not carry net power.

In this paper we study the effects of coupling between the SRRs forming the pair for the first time, neglecting the coupling between adjacent SRRs. Moreover, orthogonal orientation between the symmetry plane of the CPW and the symmetry plane of the pair of SRRs is considered. The consideration of all the effects together is extremely complex. However, for many purposes, the orthogonal orientation is the convenient one (to avoid mixed coupling) and coupling 
between adjacent (cascaded) resonators does not apply in single stage structures, or if the SRRs are distant enough. Moreover, although for the implementation of metamaterial transmission lines the pairs of SRRs are implemented with identical resonators, disruption of symmetry may be of interest in certain applications, such as sensors and comparators based on symmetry properties. Therefore, we will consider the more general case of CPWs with asymmetric SRR loading (i.e., with different SRRs forming the pair). This asymmetry, and the inclusion of magnetic coupling between the resonators forming the pair is the novel aspect of the present paper as compared to previous models. In section II, we present the circuit model of these SRR-loaded lines, including magnetic coupling between the SRRs forming the pair. From this model we will be able to obtain the SRR resonances (or zeros in the transmission coefficient), where different situations will be considered: symmetric loading (identical SRRs), asymmetric loading with identical resonance frequencies of the individual SRRs but different reactance slope, and asymmetric loading with different resonance frequencies of the individual SRRs. The model is then validated in section III through parameter extraction. Finally, the main conclusions are highlighted in section IV.

\section{CIRCUIT MODEL AND ANALYSIS}

A typical topology of the considered lines (unit cell), with identical SRRs, is depicted in Fig. 1(a). Rupture of symmetry can be achieved, for instance, by using different SRRs, or by loading the SRRs with asymmetric dielectric loads (interesting for dielectric characterization by comparison to a reference load). The circuit model of the unit cell of these lines, that accounts for both magnetic couplings (line-toresonator, through $M_{1}$ and $M_{2}$, and inter-resonator coupling, through $M^{\prime}$ ), is depicted in Fig. 1(b). $L$ and $C$ are the per-unitcell inductance and capacitance of the line, and the SRRs are described by the resonant tanks $L_{1}-C_{1}$ and $L_{2}-C_{2}$.

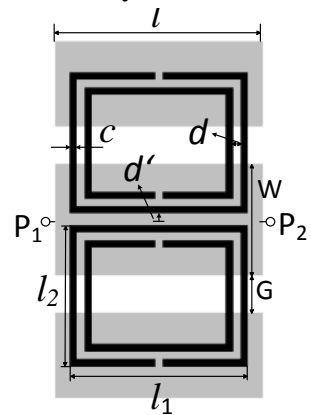

(a)

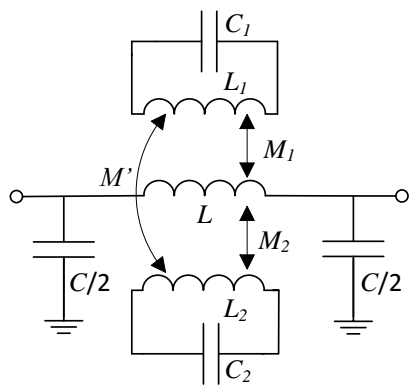

(b)
Fig. 1. Typical topology of a CPW loaded with a pair of SRRs (a), and lumped element equivalent circuit model, considering magnetic coupling between SRRs (b). The SRRs (in black) are etched in the back substrate side of the CPW (depicted in grey). The model considers the general case of different SRRs, or identical SRRs with different dielectric loading.

The circuit of Fig. 1(b) is a $\pi$-circuit where the shunt branches are purely capacitive. Thus, the transmission zeros (or resonance frequencies of the coupled resonators) are given by the poles of the series reactance. From Kirchhoff's equations, including the different mutual couplings, applied to the series branch of the circuit of Fig. 1(b), the impedance of this branch is found to be:

$$
\times\left\{\frac{Z_{s}(\omega)=j \omega L+j \omega^{3} \times}{\left(1-\frac{\omega^{2}}{\omega_{1}^{2}}\right)\left(1-\frac{\omega^{2}}{\omega_{2}^{2}}\right)-\omega^{4} M^{\prime 2} C_{1} C_{2}}\right\}
$$

where $\omega_{1}=\left(L_{1} C_{1}\right)^{-1 / 2}$ and $\omega_{2}=\left(L_{2} C_{2}\right)^{-1 / 2}$ are the resonance frequencies of the isolated resonators. By forcing the denominator of the last term to be zero, the notch (or transmission zero) frequencies can be derived, namely:

$$
\omega_{ \pm}^{2}=\frac{\omega_{1}^{2}+\omega_{2}^{2} \pm \sqrt{\left(\omega_{1}^{2}-\omega_{2}^{2}\right)^{2}+4 M^{\prime 2} \omega_{1}^{4} \omega_{2}^{4} C_{1} C_{2}}}{2\left[1-M^{\prime 2} \omega_{1}^{2} \omega_{2}^{2} C_{1} C_{2}\right]}
$$

Note that these frequencies do not depend on the mutual couplings, $M_{1}$ and $M_{2}$, between the line and the SRRs, but on the mutual coupling, $M^{\prime}$, between the SRRs. If inter-resonator coupling is negligible $\left(M^{\prime}=0\right)$, reasonable if the SRRs are separated enough, the solutions of (2) are simply $\omega_{1}$ and $\omega_{2}$. In this case, the equivalent circuit is formally identical to the one reported in [16], but with two different resonators in the series branch, due to the asymmetry of the unit cell. Let us now discuss three specific situations considering that $M^{\prime} \neq 0$.

\section{A. Symmetric case}

The simplest one is the symmetric case, where $M_{1}=M_{2}=$ $M, \omega_{1}=\omega_{2}=\omega_{0}, L_{1}=L_{2}=L_{r}$, and $C_{1}=C_{2}=C_{r}$. Under these conditions, the two solutions of (2) are $\left(k_{M}\right.$ is the magnetic coupling coefficient):

$$
\omega_{ \pm}=\frac{\omega_{o}}{\sqrt{1 \mp \frac{M^{\prime}}{L_{r}}}}=\frac{\omega_{o}}{\sqrt{1 \mp k_{M}}}
$$

However, one of the solutions, the one with the (-) sign in the radicand of (3) $\left(\omega_{+}\right)$, is not actually a transmission zero frequency. The reason is that this frequency also nulls the numerator of the last term in (1). By applying l'Hôpital's rule, it follows that the series impedance is finite at the frequency $\omega_{+}$; hence, we can conclude that only one transmission zero, to the left of $\omega_{0}$ (since $M^{\prime}>0$ ), appears in the transmission coefficient for symmetric structures with magnetically coupled SRRs.

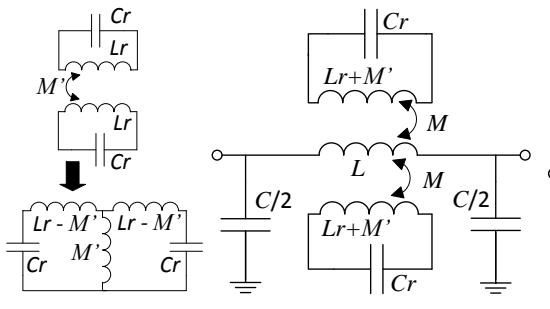

(a)

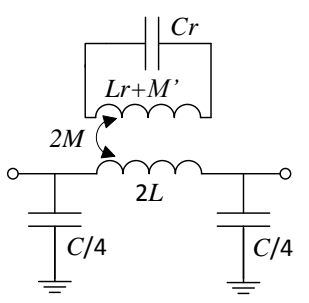

(b)
Fig. 2. Circuit model of the structure of Fig. 1 for the symmetric case, with the inductive coupling between resonators transformed to the equivalent $\mathrm{T}$ model (a), and circuit that results by applying the magnetic wall concept (b).

The transmission zero frequency for the symmetric case, $\omega_{-}$, can alternatively be obtained by applying the magnetic wall concept to the circuit of Fig. 1(b). Before that, it is necessary to transform the magnetically coupled inductors $L_{r}$ 's of the 
circuit of Fig. 1(b) to the equivalent T-circuit model (see Fig. 2) [21]. Notice that the inductance of each resonator at both sides of the symmetry plane (magnetic wall) is given by $L_{r}+M^{\prime}$, from which expression (3) with the (+) sign results. For the symmetric case, the model of [13],[16] provides the same results, but according to this new analysis the equivalent inductance of the resonators includes the effects of $M^{\prime}$, i.e., in [13],[16] $M^{\prime}$ is included in the SRR inductance.

\section{B. Asymmetric case (different SRRs with same resonance)}

The next analysis is for the asymmetric structure (different coupled SRRs), but considering that their fundamental resonance frequencies are identical, that is, $L_{1} \neq L_{2}, C_{l} \neq C_{2}$, $\omega_{1}=\omega_{2}=\omega_{0}$. In this case, expression (2) gives:

$$
\omega_{ \pm}=\frac{\omega_{o}}{\sqrt{1 \mp \frac{M^{\prime}}{\sqrt{L_{1} L_{2}}}}}=\frac{\omega_{o}}{\sqrt{1 \mp k_{M}}}
$$

Note that (4) is formally identical to (3), but replacing the inductance $L_{r}$ with the geometrical mean of the two inductances $L_{1}$ and $L_{2}$ (both expressions can be written in terms of the magnetic coupling coefficient, $k_{M}$ ). The two frequencies given by (4) null the denominator of the last term in (1), but, in general, none of them nulls the numerator. However, if the following condition is satisfied

$$
C_{1} M_{1}^{2}+C_{2} M_{2}^{2}=2 M_{1} M_{2} \sqrt{C_{1} C_{2}}
$$

then the numerator of the last term in (1) is also null at $\omega_{+}$, and the impedance of the series branch is finite at this frequency (as the application of l'Hôpital's rule reveals). Expression (5) can be simplified to:

$$
\frac{M_{1}}{M_{2}}=\sqrt{\frac{L_{1}}{L_{2}}}=\sqrt{\frac{C_{2}}{C_{1}}}
$$

Thus, if condition (6) is satisfied, then only one transmission zero (at $\omega_{-}$) is expected (notice that (6) represents a balance that forces the structure to behave similarly to the symmetric one). Otherwise, two transmission zeros at the frequencies given by (4), i.e., one above and the other below $\omega_{0}$, are expected.

\section{General case}

The last analysis corresponds to the asymmetric case with arbitrary resonator frequencies $\left(L_{1} \neq L_{2}, C_{1} \neq C_{2}, \omega_{1} \neq \omega_{2}\right)$. In this case, the transmission zeros are given by the two solutions of (2), and the mutual coupling between resonators $\left(M^{\prime}\right)$ enhances the distance between the transmission zeros, i.e.,

$$
\omega_{+}^{2}-\omega_{-}^{2}=\frac{\sqrt{\left(\omega_{1}^{2}-\omega_{2}^{2}\right)^{2}+4 M^{\prime 2} \omega_{1}^{4} \omega_{2}^{4} C_{1} C_{2}}}{\left[1-M^{\prime 2} \omega_{1}^{2} \omega_{2}^{2} C_{1} C_{2}\right]}>\omega_{1}^{2}-\omega_{2}^{2}
$$

If the resonance frequencies of the isolated resonators $\left(\omega_{1}\right.$ and $\left.\omega_{2}\right)$ are distant enough $\left(\left|\omega_{1}{ }^{2}-\omega_{2}{ }^{2}\right| \gg 0\right)$ and $M^{\prime}$ is small (this later condition is very reasonable in the considered structures), the two solutions of (2) can be approximated by:

$$
\omega_{+}=\omega_{1} \sqrt{\frac{1+R \cdot k_{M}^{2}}{1-k_{M}^{2}}}>\omega_{1} ; \omega_{-}=\omega_{2} \sqrt{\frac{1-(1+R) \cdot k_{M}^{2}}{1-k_{M}^{2}}}<\omega_{2}
$$

where we have considered that $\omega_{1}>\omega_{2}$, and the positive dimensionless factor $R=\left(\omega_{1}^{2} / \omega_{2}^{2}-1\right)^{-1}$ has been introduced to simplify expressions (8). The validity of (8) is subjected to large values of $\omega_{1}^{2}-\omega_{2}^{2}$ (small $R$ ) and small values of $M^{\prime}$ (or $k_{M}$ ), avoiding negative radicand in the square root of $\omega_{-}$in (8).

\section{VALIDATION}

To validate the proposed model, we first consider the symmetric case, where SRR and CPW dimensions are set to $l_{1}$ $=4.8 \mathrm{~mm}, l_{2}=3.8 \mathrm{~mm}, c=d=0.2 \mathrm{~mm}, l=5.6 \mathrm{~mm}, W=3 \mathrm{~mm}$, and $G=1.01 \mathrm{~mm}$. The lossless electromagnetic simulations (inferred from Agilent Momentum) of the structure $\left(S_{2 I}\right)$, considering different values of inter-resonator distance, $d$ ', are depicted in Fig. 3 (the considered substrate is Rogers RO3010 with dielectric constant $\varepsilon_{r}=11.2$ and thickness $h=1.27 \mathrm{~mm}$ ). The circuit parameters for the four considered cases (extracted from the method reported in [22]) are shown in Table I (analytical models for the SRRs are reported in [12], [23],[24], but these models are not accurate enough with the presence of the $\mathrm{CPW}$ ). As $d$ ' increases, the mutual coupling $M^{\prime}$ decreases, and the resonance frequency increases. Note that the other circuit parameters do not significantly vary, and the agreement between circuit and electromagnetic simulations in the region of interest is very good, pointing out the validity of the model. The measured responses (shown in Fig. 3) are also in good agreement (slight discrepancies are due to losses and fabrication related tolerances).

\section{TABLE I. EXTRACTED CIRCUIT PARAMETERS (SYMMETRIC} CASE) FOR DIFFERENT VALUES OF $d$,

\begin{tabular}{|c|c|c|c|c|c|c|}
\hline $\boldsymbol{d}^{\prime}(\mathbf{m m})$ & $\boldsymbol{L}(\mathbf{n H})$ & $\boldsymbol{C}(\mathbf{p F})$ & $\boldsymbol{C}_{\boldsymbol{r}}(\mathbf{p F})$ & $\boldsymbol{L}_{\boldsymbol{r}}(\mathbf{n H})$ & $\boldsymbol{M}(\mathbf{n H})$ & $\boldsymbol{M}^{\prime}(\mathbf{n H})$ \\
\hline 0.105 & 1.82 & 1.58 & 0.44 & 6.85 & 0.82 & 1.74 \\
\hline 0.305 & 1.86 & 1.58 & 0.44 & 6.85 & 0.82 & 1.29 \\
\hline 0.505 & 1.84 & 1.57 & 0.43 & 6.85 & 0.81 & 1.02 \\
\hline 0.755 & 1.85 & 1.55 & 0.43 & 6.85 & 0.80 & 0.80 \\
\hline
\end{tabular}

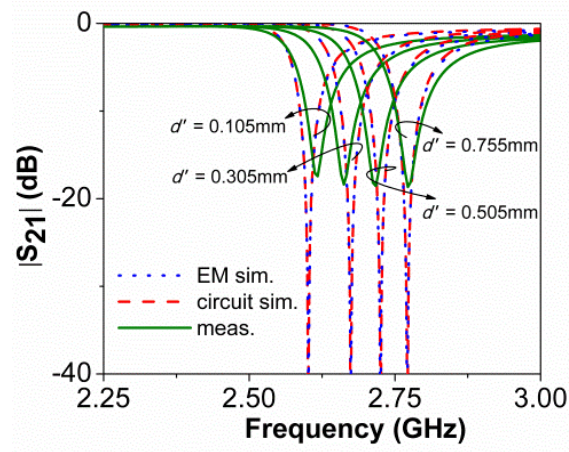

(a)

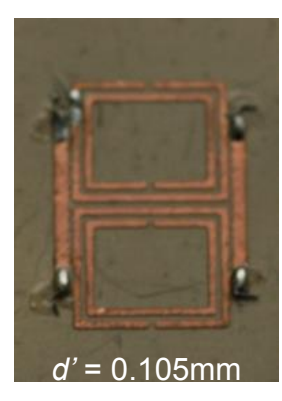

(b)
Fig. 3. Frequency response (symmetric case) for different values of $d^{\prime}$ (a), and detail of one of the fabricated samples (bottom) where the ground planes have been connected through vias and strips to avoid the slot mode (b).

Let us now consider the asymmetric SRR-loaded line with identical resonance frequency for both SRRs $\left(\omega_{1}=\omega_{2}=\omega_{0}\right)$. The considered geometrical parameters are: $l=5.6 \mathrm{~mm}, W=$ $3 \mathrm{~mm}, G=1.01 \mathrm{~mm}$; upper (smaller) SRR: $l_{1}=4 \mathrm{~mm}, l_{2}=$ $3 \mathrm{~mm}, c=0.2 \mathrm{~mm}, d=0.1 \mathrm{~mm}, d^{\prime}=0.305 \mathrm{~mm}$; lower (larger) SRR: $l_{1}=4.5 \mathrm{~mm}, l_{2}=4.1 \mathrm{~mm}, c=0.2 \mathrm{~mm}, d=0.725 \mathrm{~mm}, d^{\prime}=$ $0.155 \mathrm{~mm}$. The extracted parameters are: $L=1.77 \mathrm{nH}, C=$ $1.6 \mathrm{pF}, L_{l}=5.0 \mathrm{nH}, C_{l}=0.42 \mathrm{pF}, L_{2}=7.2 \mathrm{nH}, C_{2}=0.29 \mathrm{pF}, M_{I}$ $=0.513 \mathrm{nH}, M_{2}=0.774 \mathrm{nH}, M^{\prime}=0.96 \mathrm{nH}$. As expected, two notches (one above and the other below $\omega_{0}$ ) are present (the agreement between the circuit and electromagnetic simulation 
is very good, according to Fig. 4). We have verified through circuit simulation that when condition (6) is satisfied, only one notch appears (see Fig. 4).

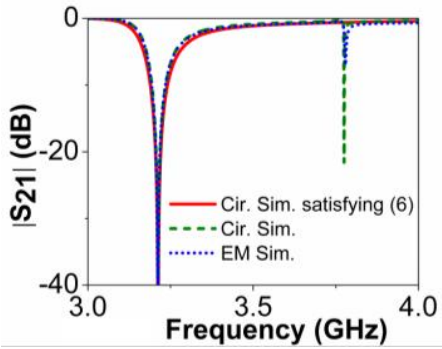

(a)

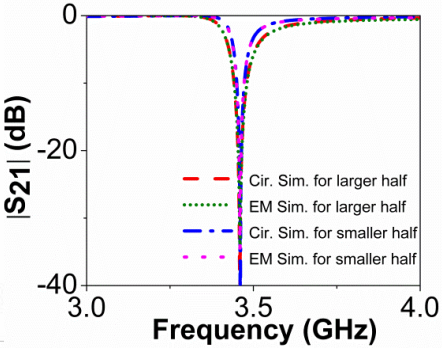

(b)
Fig. 4. (a) Frequency response for the considered asymmetric case with identical SRRs resonance frequencies, and circuit simulation corresponding to $L_{l}=5 \mathrm{nH}, L_{2}=7.2 \mathrm{nH}, M_{2}=0.774 \mathrm{nH}, M_{1}=0.645 \mathrm{nH}$, i.e., satisfying (6); (b) responses of the CPW loaded with only the larger or smaller SRR.

Finally, we have considered an asymmetric structure with different SRR resonance frequencies. The geometry is as follows: $l=5.6 \mathrm{~mm}, W=3 \mathrm{~mm}, G=1.01 \mathrm{~mm}$; upper SRR: $l_{1}=$ $4.8 \mathrm{~mm}, l_{2}=4.6 \mathrm{~mm}, c=d=0.2 \mathrm{~mm}$; lower SRRs: $l_{1}=4.8 \mathrm{~mm}$, $l_{2}=3.8 \mathrm{~mm}, c=d=0.2 \mathrm{~mm}$. We have obtained the frequency response and the circuit parameters for four different values of $d$ '. The pair of notches (at $f_{-}=\omega / 2 \pi$ and $f_{+}=\omega_{+} / 2 \pi$ ) are depicted in Fig. 5, and verify that their distance increases as $d^{\prime}$ decreases, and $f_{-}<f_{2}$ and $f_{+}>f_{1}$, in agreement to (8).

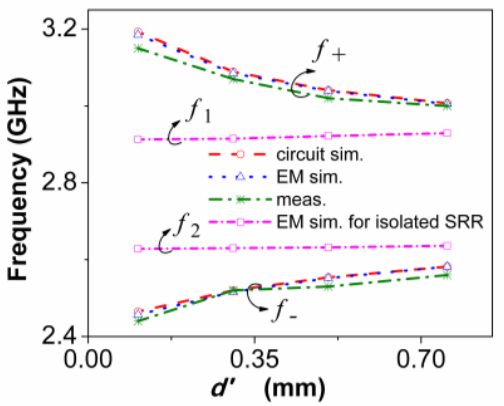

(a)

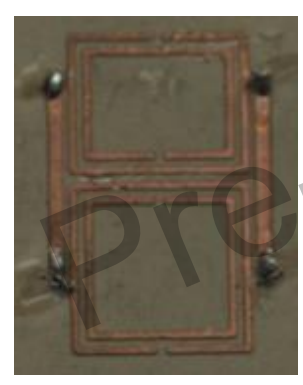

(b)
Fig. 5. Variation of the notch frequencies as a function of $d^{\prime}$ for the asymmetric SRR-loaded CPW (a), and detail of the fabricated sample (bottom face) for $d^{\prime}=0.105 \mathrm{~mm}$ (b)

\section{CONCLUSION}

We have proposed and validated a circuit model for CPWs loaded with pairs of magnetically coupled SRRs. The model is valid for both symmetric and asymmetric structures. In the former case, only one notch in the transmission coefficient appears. For asymmetric structures, two notches are present and their separation increases as the distance between resonators decreases. However, there is a particular case of asymmetry, representing a balance condition between circuit parameters, where only one notch appears. The agreement between circuit, electromagnetic simulations and experiment is good in all the cases, supporting the validity of the model.

\section{REFERENCES}

[1] M. Durán-Sindreu, A. Vélez, G. Sisó, J. Selga, P. Vélez, J. Bonache, and F. Martín "Recent advances in metamaterial transmission lines based on split rings", Proc. IEEE, vol. 99, pp. 1701-1710, Oct. 2011.

[2] H. Okabe, C. Caloz, T. Itoh, "A compact enhanced bandwidth hybrid ring using an artificial lumped element left handed transmission line section", IEEE Trans. Microw. Theory Techn., vol. 52, pp. 798-804, Mar. 2004.
[3] M.A. Antoniades and G.V. Eleftheriades, "A broadband series power divider using zero-degree metamaterial phase shifting lines", IEEE Microw. Wirel. Comp. Lett., vol. 15, pp. 808-810, Nov. 2005.

[4] M.A. Antoniades, and G.V. Eleftheriades, "A broadband Wilkinson balun using microstrip metamaterial lines", IEEE Ant. Wirel. Propag. Lett., vol. 4, pp. 209-212, 2005.

[5] G. Sisó, J. Bonache, M. Gil and F. Martín, "Application of resonanttype metamaterial transmission lines to the design of enhanced bandwidth components with compact dimensions", Microw. Opt. Techn. Lett., vol. 50, pp. 127-134, Jan. 2008.

[6] I-H. Lin, M. DeVincentis, C. Caloz, and T. Itoh, "Arbitrary dual-band components using composite right/left-handed transmission lines", IEEE Trans. Microw. Theory Techn., vol. 52, pp. 1142-1149, Apr. 2004.

[7] A.C. Papanastasiou, G.E. Georghiou, G.V. Eleftheriades, "A quad-band wilkinson power divider using generalized NRI transmission lines", IEEE Microw. Wirel. Comp. Lett., vol. 18, pp. 521-523, Aug. 2008.

[8] M. Durán-Sindreu, G. Sisó, J. Bonache, F. Martín, "Planar multi-band microwave components based on the generalized composite right/left handed transmission line concept", IEEE Trans. Microw. Theory Techn., vol. 58, no 12, pp. 3882-3891, Dec. 2010.

[9] S. Lim, C. Caloz, T. Itoh," Electronically Scanned Composite Right/Left Handed Microstrip Leaky-Wave Antenna", IEEE Microw. Wirel. Comp. Lett., vol. 14, pp. 277-279, Jun. 2004.

[10] M.A. Antoniades, G.V. Eleftheriades, "A CPS leaky-wave antenna with reduced beam squinting using NRI-TL metamaterials," IEEE Trans. Antennas Propag., vol. 56, no. 3, pp. 708-721, Mar. 2008.

[11] G. Zamora, S. Zuffanelli, F. Paredes, F. Javier Herraiz-Martínez, F. Martín, J. Bonache, "Fundamental mode leaky-wave-antenna (LWA) using slot line and split-ring-resonator (SRR) based metamaterials", IEEE Ant. Wirel. Prop. Lett., vol. 12, pp. 1424-1427, 2013.

[12] R. Marqués, F. Martín and M. Sorolla, Metamaterials with Negative Parameters: Theory, Design and Microwave Applications, John Wiley \& Sons Inc., New York, 2007.

[13] F. Martín, F. Falcone, J. Bonache, R. Marqués and M. Sorolla, "Split ring resonator based left handed coplanar waveguide", Appl. Phys. Lett., vol. 83, pp. 4652-4654, December 2003.

[14] F. Falcone, T. Lopetegi, J.D. Baena, R. Marqués, F. Martín and M. Sorolla, "Effective negative- $\varepsilon$ stop-band microstrip lines based on complementary split ring resonators", IEEE Microw. Wirel. Comp. Lett., vol. 14, pp. 280-282, Jun. 2004.

[15] J.D. Baena, J. Bonache, F. Martín, R. Marqués, F. Falcone, T. Lopetegi, M.A.G. Laso, J. García, I Gil, M. Flores-Portillo and M. Sorolla, "Equivalent circuit models for split ring resonators and complementary split rings resonators coupled to planar transmission lines", IEEE Trans. Microw. Theory Techn., vol. 53, pp. 1451-1461, Apr. 2005.

[16] F. Aznar, J. Bonache and F. Martín, "Improved circuit model for left handed lines loaded with split ring resonators", Appl. Phys. Lett., vol. 92, paper 043512, February 2008.

[17] J. Naqui, M. Durán-Sindreu, and F. Martín, "Modeling Split Ring Resonator (SRR) and Complementary Split Ring Resonator (CSRR) Loaded Transmission Lines Exhibiting Cross Polarization Effects", IEEE Ant. Wirel. Propag. Lett., vol. 12, pp. 178-181, 2013.

[18] R. R. A. Syms, and L. Solymar, "Effective permeability of a metamaterial: against conventional wisdom", App. Phys. Lett. vol. 100, paper 124103 (2012).

[19] J. Naqui, A. Fernández-Prieto, F. Mesa, F. Medina and F. Martín, "Effects of inter-resonator coupling in split ring resonator (SRR) loaded metamaterial transmission lines", J. Appl. Phys., vol. 115, paper 194903, 2014.

[20] T. Tamir and A. A. Oliner, "Guided complex waves," Proc. Inst. Elect. Eng., vol. 110, pp. 310-334, 1963.

[21] J-S. Hong and M.J. Lancaster, Microstrip filters for RF/microwave applications, John Wiley, New Jersey, 2001.

[22] F. Aznar, M. Gil, J. Bonache, J.D. Baena, L. Jelinek, R. Marqués, F. Martín, "Characterization of miniaturized metamaterial resonators coupled to planar transmission lines" J. Appl. Phys., vol. 104, paper 114501-1-8, Dec. 2008.

[23] F. Bilotti, A. Toscano, L. Vegni, K. Aydin, K. Alici, and E. Ozbay, "Equivalent-circuit models for the design of metamaterials based on artificial magnetic inclusions", IEEE Trans. Microw. Theory Techn., vol. 55 , no. 12 , pp. 2865 - 2873, Dec. 2007.

[24] D. Ramaccia, L. Di Palma, D. Ates, E. Ozbay, A. Toscano, and F. Bilotti, "Analytical model of connected bi-omega: robust particle for the selective power transmission through sub-wavelength apertures", IEEE Trans. Ant. Prop., vol. 62, no. 4, pp. 2093-2101, Apr. 2014. 